\title{
Conocimientos locales y desarrollo sostenible en Perú. El proyecto SIPAM Agrosistemas de alta montaña
}

Beatriz Pérez Galán | Dpto. de Antropología Social y Cultural, Universidad Nacional de Educación a Distancia (UNED)

URL de la contribución <www.iaph.es/revistaph/index.php/revistaph/article/view/4949>

\section{RESUMEN}

En esta contribución se reflexiona sobre las bases sociales y culturales de los sistemas agrícolas tradicionales en el caso de los pueblos indígenas al sur de los andes peruanos. El ejemplo se extrae del proyecto piloto Agroecosistemas de alta montaña de Machupichhu al lago Titicaca, integrado al programa SIPAM (FAO-GEF). Desde una perspectiva antropológica crítica y con el énfasis puesto en la matriz cultural de las prácticas agrícolas tradicionales indígenas, en este texto se analiza la concepción estática, reificada, homogénea y esencialista que es atribuida en la práctica por el discurso conservacionista y del desarrollo sostenible a los conocimientos locales indígenas en este proyecto, lo que dificultaría en la práctica su conservación.

\section{Palabras clave}

Conocimientos locales | Desarrollo sostenible | Los Andes | Patrimonio agrícola | Pueblos indígenas | SIPAM | Perú | 


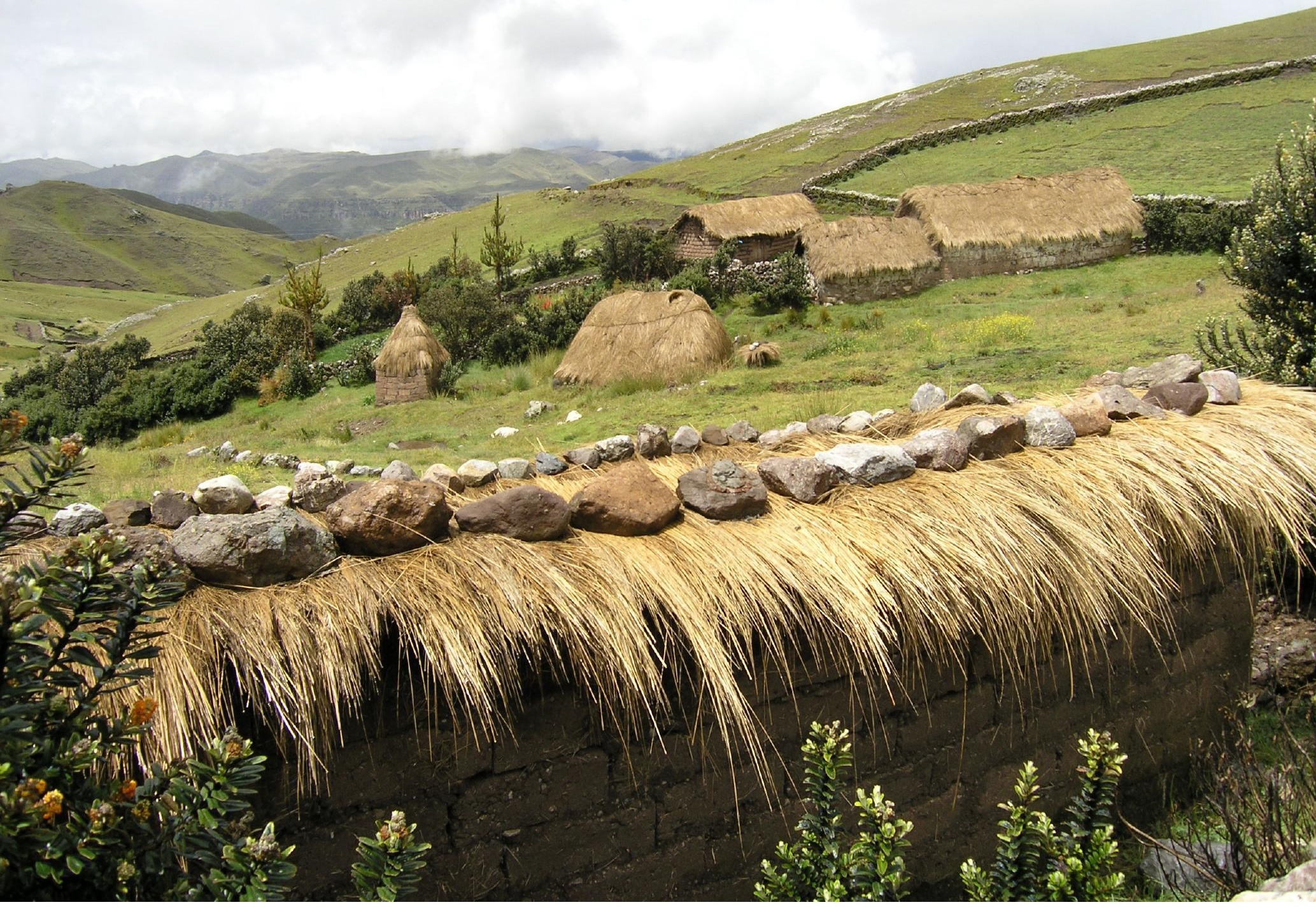

Casas tradicionales. Distrito de Accha, Paruro, Cuzco | foto Beatriz Pérez Galán, autora de todas las imágenes que ilustran este artículo, salvo que se indique lo contrario 
Una versión previa de este texto fue publicada en 2015 (Pérez Galán, 2015) en el marco del proyecto de investigación El patrimonio agrario. La construcción cultural del territorio a través de la actividad agraria financiado por el Ministerio de Ciencia e Innovación, Plan Nacional de I+D+i, 2011-14.



Fila india de autoridades mujeres o wachu, Pisac, Cuzco

\section{BASES SOCIOCULTURALES DE LOS SISTEMAS AGRÍCOLAS TRADICIONALES ANDINOS ${ }^{1}$}

En la literatura antropológica existen numerosos ejemplos en los que se analizan metáforas mediante las cuales concebimos y ordenamos simbólicamente nuestra experiencia cotidiana en categorías culturales (Lakoff y Johnson 1995). En el caso de los pueblos y comunidades indígenas de los Andes las analogías más habitualmente utilizadas proceden de los elementos de una naturaleza profundamente sacralizada, de los animales y, de forma singular, de las prácticas agrícolas. Concretamente, la imagen que emplean los indígenas quechuas del valle del río Vilcanota en el Dpto. de Cuzco para referirse a un tipo de ordenamiento normativo social, cosmológico y político es el wachu o surco de la siembra más profundo y con lomos más altos de lo habitual. Otras técnicas tradicionales del trabajo agrícola son los campos elevados (camellones o suqakollo) y las terrazas de cultivo (andenes, bancales o pata-pata), que constituyen algunos de los paisajes más emblemáticos de América Latina.

En concreto, la metáfora del surco de la siembra se emplea actualmente por esta población para designar un conjunto amplio de prácticas culturales relativas a la organización social, económica, política y religiosa. Entre los ejemplos más evidentes del paralelismo que los runas (seres humanos) establecen entre uno y otro campo semántico podemos mencionar: la alternancia de ciclos de cultivos y descanso de las parcelas, y su obligación de atender por turno los trabajos comunitarios; entre los sistemas de trabajo agrícola basados en la reciprocidad (ayni, minka), y la serie de derechos y obligaciones que estructuran su sistema de autoridades tradicionales; entre la forma de cultivar la tierra en parejas warmi-qari (varón-mujer), y la manera en que son asumidos y realizados los cargos de representación comunal; y quizás, el más evidente de todos ellos: entre la distribución lineal de las semillas en el surco de la siembra o wachu, propiamente dicho, y la colocación también lineal en cualquier faena comunal o evento festivo-religioso de las autoridades tradicionales, cuya cabeza visible son los alcaldes envarados (varayoq) (Pérez Galán 2004).

Este breve ejemplo etnográfico ilustra además la continuidad que las cosmovisiones indígenas establecen entre el mundo biofísico, el humano y el sobrenatural como parte de una misma comunidad social integrada por animales, plantas y el resto de seres que habitan el entorno natural. La antropología (Ploeg 1990; Escobar 1999; Descola 2001; Viveiros de Castro 2013), la etnoecología (Toledo 1992), y la agroecología (Hecht 1999; Altieri 1999) son algunas de las disciplinas que apuestan por superar la dicotomía naturaleza-cultura característica del pensamiento occidental y considerar las bases socioculturales de las prácticas agrícolas y los sistemas de conocimiento local en que estas se sustentan. Desde esa perspectiva, el valor cultural 


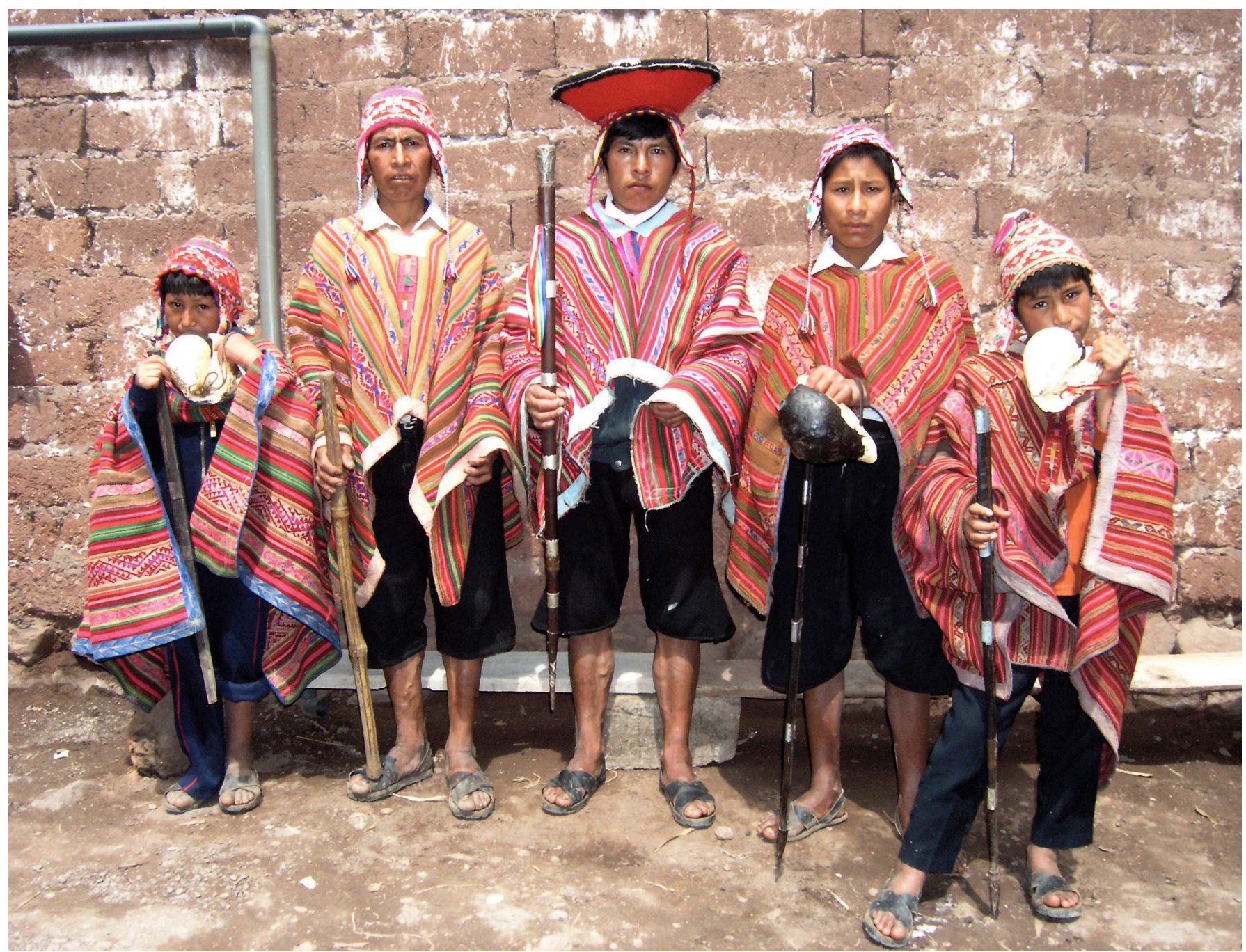

Fila india o wachu de las autoridades tradicionales de vara. Pisac, Cuzco

de los sistemas agrícolas tradicionales indígenas residiría no tanto en su asociación a un entorno natural extraordinario que es necesario conservar (parques naturales y arqueológicos, reservas de la biosfera o centros de diversidad genética), sino además por constituir un conglomerado de significados a la vez ordenados y ordenadores de la cosmología del grupo que transmite un modo de vida de la gente que habita ese territorio (Cosgrove 1984; Connerton 1989; Küchler 1993; Hirsch 1995).

Con el énfasis puesto en la matriz cultural de los sistemas agrícolas, en este texto entendemos el conocimiento de las poblaciones locales como una actividad práctica sociocultural, situada y cambiante. Siguiendo a distintos autores (Ploeg 1990; Warren, Slikkerveer y Brokensha 1995; Reyes-García 2007), estas formas de conocimiento se caracterizarían por su alto grado de 
Impulsado en 2002 por la Organización de las Naciones Unidas para la Agricultura y la Alimentación (FAO), los SIPAM (GIAHS, siglas en inglés) son definidos como "sistemas de uso de la tierra y paisajes extraordinarios ricos en diversidad biológica de importancia mundial, evolucionando desde la co-adaptación de una comunidad con su ambiente y sus ne cesidades y aspiraciones para un desarrollo sostenible" (Koohafkan y Altieri 2010, 1)

\section{3}

Entre los antecedentes directos del SIPAM peruano, se incluyen: el Proyecto Conservación in situ de Cultivos Nativos y de sus Parientes Silvestres (2001-06), una de las experiencias más completas en ese ámbito coordinada de varias organizaciones peruanas públicas y privadas dedicadas a la conservación (ARARIWA, CCTA, CESA, IIAP, INIA y PRATEC) Otras iniciativas de conservación menos ambiciosas y con resultados dispares han sido promovidas por el Servicio Silvo Agropecuario (SESA), la ONG Jorge Basadre, ASPADERUC, CONDESAN, y el proyecto Conserva ción in situ de Raíces y Tuberosas Andinas, ejecutado por el PRONIRGEB-INIA. En e sector de las ONGs destacan CIED, IDEAS CEDEP, DESCO, PISCA, e IDEMA, la aso ciación Andes-Parque de la Papa (Pisac), la Red de Agricultura Ecológica, y la Asociación Nacional de Productores Ecológicos (ANPE).

4

El Fondo Mundial para el Medio Ambiente o GEF (en sus siglas en inglés) es una asociación global integrada por 178 países, instituciones internacionales, organizaciones no gubernamentales y el sector privado. Creada en 1991 como un programa específico del Banco Mundial para la protección del medio ambiente y el desarrollo sostenible, se reestructura en 1994 como institución independiente para convertirse en un mecanismo financiero de las convenciones de Río. Más información en: https://www.thegef.org/project/cbpf-conservation-and-adaptive-management-globally-important-agricultural-heritage-systems [Consulta: $28 / 05 / 2021]$ heterogeneidad, por ser subjetivas (dependientes de sujetos que lo significan), por transmitirse oralmente, por su origen híbrido (mezcla de tradiciones de diversa procedencia reapropiadas históricamente), por su recurrencia a la "tradición" como criterio de autoridad, y por formar parte de un todo que está integrado práctica y conceptualmente en sistemas de valores y creencias (cosmovisión). Esta concepción antropológica se aleja de la naturaleza estática, reificada, homogénea, aborigen y ancestral atribuida frecuentemente por el discurso conservacionista y del desarrollo sostenible a los conocimientos locales que dificulta en la práctica su conservación (Erickson 2006; Herrera 2013; Pérez Galán 2015).

Para ilustrar esta idea, el ejemplo etnográfico procede del proyecto piloto Agroecosistemas de alta montaña de Machupicchu al Lago Titicaca, integrado en el programa Sistemas Importantes del Patrimonio Agrícola Mundial² (FAO 2008; Koohafkan y Altieri 2010). En este territorio se concentra una enorme variedad de especies endémicas (papas, maíces, quinoas y frutales nativos), crianzas nativas (alpacas), y el uso de tecnologías tradicionales agrícolas como los wachus, los andenes, los campos elevados y la cosecha del agua de lluvia en lagos artificiales, muchas en desuso en la actualidad. Para paliar las amenazas que afectan a este territorio como la migración a la ciudad, la modernización tecnológica, el cambio climático, la erosión, la pérdida de la biodiversidad asociada y las barreras de la competencia con alimentos importados y subsidiados, el SIPAM peruano se une a otras iniciativas de conservación in situ de la biodiversidad que en las últimas dos décadas han tratado de conservar y poner en valor los sistemas agrícolas tradicionales en la región ${ }^{3}$. En particular, como antropóloga, en esta oportunidad me interesa explorar el papel otorgado de facto en este proyecto a la población local y cuál es la contribución efectiva de sus formas de organización socioeconómicas y políticas y de sus conocimientos locales sobre sistemas de cultivo, tecnologías agrícolas y manejo del suelo, aspectos que distinguirían sobre el papel al programa SIPAM de otros (Howard et ál. 2008; Koohafkan y Altieri 2010).

\section{EL SIPAM PERUANO}

El proyecto SIPAM-Perú es resultado de un largo proceso de gestación que se remonta al año 2003 cuando reconocidos expertos peruanos en agrobiodiversidad y tecnologías agrícolas andinas se unen para elaborar una propuesta que combinase los conocimientos tradicionales agrícolas andinos y la innovación tecnológica. En 2004 esta propuesta se eleva a la FAO y a GEF4 a través del Consejo Nacional del Ambiente (CONAM), entonces el órgano gestor del Ministerio del Ambiente (MINAM) en materia de política ambiental y patrimonio natural en el país. Este proyecto contará con la colaboración del entonces Ministerio de Agricultura (MINAG) a través del Instituto Nacional de 


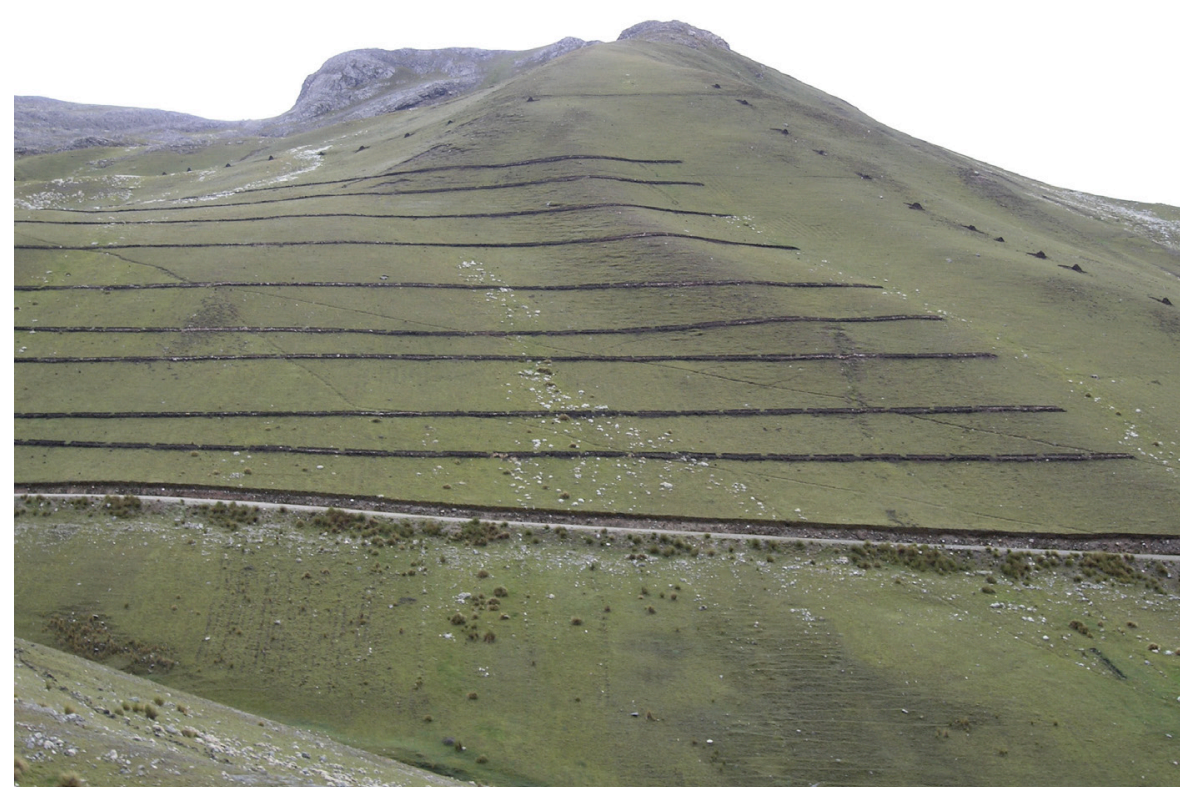

Investigación Agraria y del Proyecto "Corredor Puno-Cusco" (CONAM 2006, 2007).

Una vez seleccionado como uno de los seis sitios piloto donde poner en práctica las tesis de la conservación dinámica del patrimonio agrícola que propone el programa SIPAM, se inicia un tortuoso proceso de planificación, ajustes presupuestarios y programáticos que retrasan más de seis años el comienzo de actividades. Como resultado de estos ajustes, el proyecto originalmente diseñado para cinco años y con un presupuesto de casi dos millones de dólares (USA) queda reducido a algo menos de un tercio (600.000\$), la mitad del tiempo (de enero de 2011 a junio de 2013), y a un equipo formado por solo dos coordinadores. Ellos serán los encargados de abarcar el trabajo en 17 comunidades -unas 2500 familias-, pertenecientes a dos grupos étnicos -quechuas y aymaras-, dispersas en un área de $350 \mathrm{~km}$ (distritos de Lamay y Lares en Cuzco, y San José y Ácora en Puno), con una deficiente conectividad, sin técnicos de campo, sin oficinas y sin vehículo propio, circunstancias que lastrarán en gran medida los resultados de este proyecto.

La selección de este vasto territorio responde a un conjunto de criterios combinados. Además de poseer una excelsa biodiversidad seriamente amenazada, estas comunidades se diferencian de sus vecinas en dos aspectos clave: por un lado, la existencia de un capital social en la población de las comunidades que deriva de la experiencia previa acumulada por su participación en proyectos de recuperación de tecnologías indígenas coordinados por los mismos técnicos ${ }^{5}$. Y por otro, por el emplazamiento del proyecto
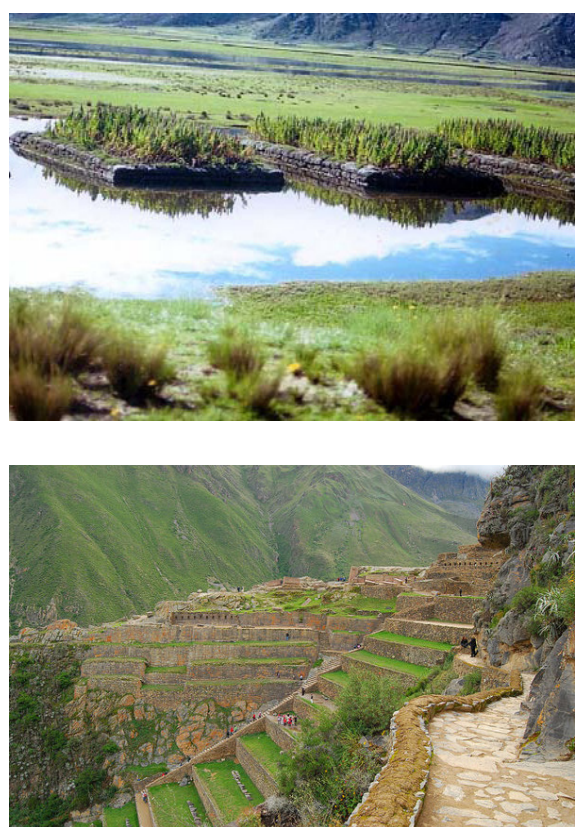

A la iaquierda, andenes o terrazas de cultivo. Accha, Paruro, Cuzco

Arriba, sukaqollos o camellones. Comunidad de Caritamaya, Ácora, Puno | fuente CONAM 2007, 22

Abajo, pata-pata o terrazas de cultivo. Parque arqueológico de Ollantaytambo, Urubamba, Cuzco

Desde finales de la década de 1980 se implementan en este territorio varios proyectos orientados a la recuperación de tecnologías, cultivos y crianzas autóctonas: Proyecto de Investigación de los Sistemas Agropecuarios Andinos (PISA), Proyecto de Investigación de los Sistemas de Cultivos Andinos (PISCA), Programa Interinstitucional de Waru-Waru (PIWA), y Programa Conservación in situ de Cultivos Nativos y de sus Parientes Silvestres. Ver nota 3. 


\section{PROYECTO SIPAM}

\section{MAPA DE UBICACION DEL PROYECTO EN PERU}

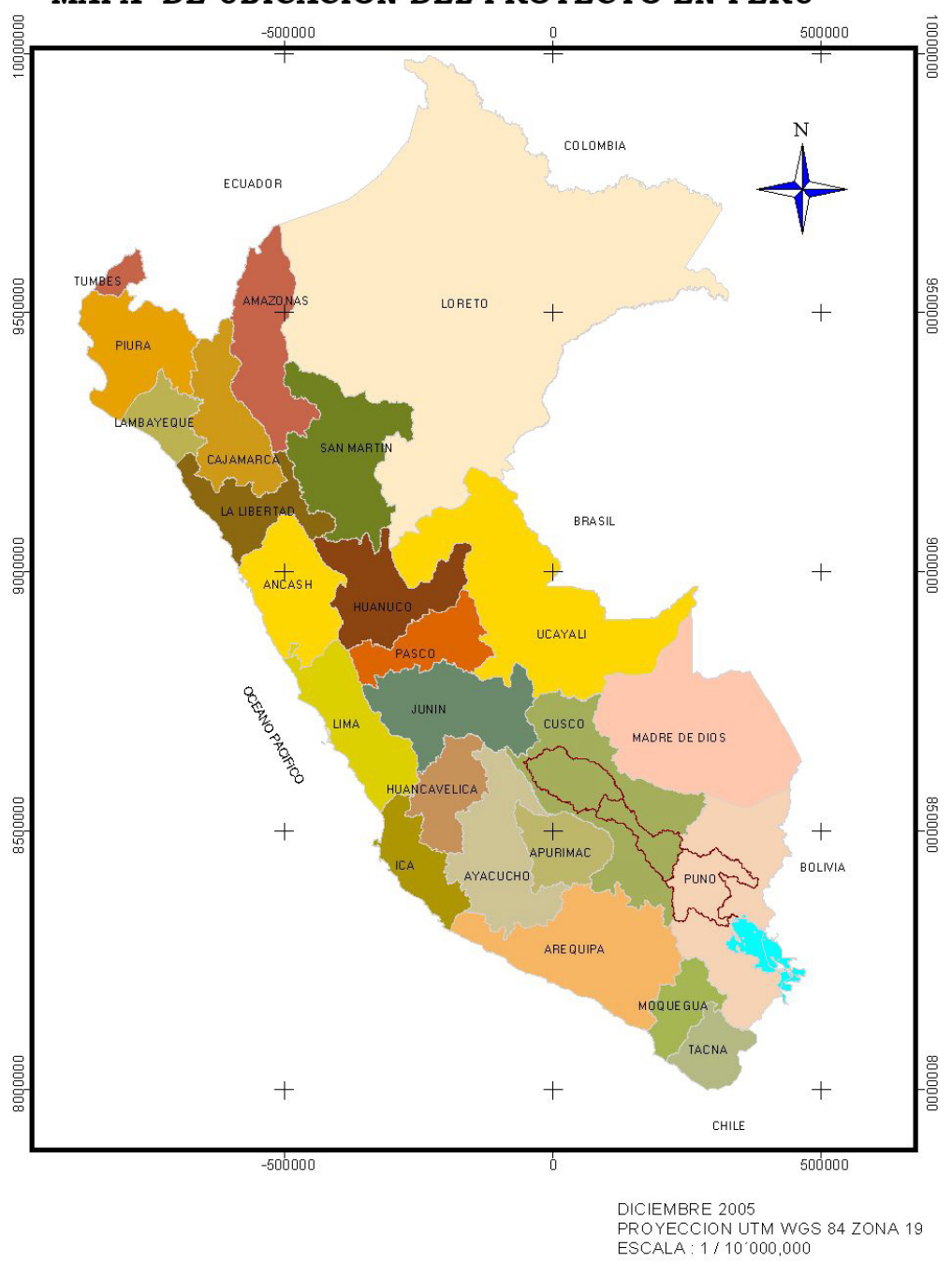

Mapa de ubicación del proyecto en el Perú | fuente CONAM 2007 en una de las rutas turísticas de gran belleza paisajista y más reconocidas internacionalmente de donde extraerá su nombre: el Santuario Histórico de Machu Picchu, integrante de la lista de Patrimonio Cultural y Natural de la Humanidad desde 1983, y el lago Titicaca, candidato a ese reconocimiento desde 2006.

Al igual que en otros casos del mismo programa (Howard et ál. 2008), la adscripción técnica y operativa del SIPAM peruano recae en la Dirección de Diversidad Biológica del Ministerio del Ambiente (MINAM), lo que tendrá importantes consecuencias. En parte, estas consecuencias derivan de la tensión no resuelta entre "conservación" (MINAM), enfocada a la protección 
del medio natural per se, versus "productividad" (MINAG), más preocupada a garantizar la seguridad alimentaria y un mayor acceso a los mercados, asuntos que por lo general son de mayor interés para la población campesina. Esa fractura entre actividad productiva y conservación ambiental que se superpone a la atribuida entre naturaleza y cultura es reproducida en la política de protección de los organismos internacionales y sería, en parte, causante de la escasa valoración real con que cuenta este patrimonio. Pues, como recuerda Castillo $(2013,17)$, aunque las prácticas agrícolas y ganaderas sean actividades claramente antropogénicas y dinámicas, los reconocimientos más importantes de que han sido objeto a nivel internacional están relacionados de forma abrumadora con lo medioambiental (es decir los efectos sobre el medio de dicha actividad) y no tanto la actividad en sí misma y los aspectos sociales y culturales en los que es preciso considerar a los sistemas agrícolas (Pérez Galán 2012; Herrera Wassilowsky 2013).

\section{OBJETIVOS, METODOLOGÍA Y RESULTADOS DEL PROYECTO}

"Las actividades productivas agrícola y ganadera involucran al $100 \%$ de la población económicamente activa de las comunidades quechuas y aymaras de los sitios piloto, lo cual implica que toda la población está sumamente interesada en acciones orientadas a la recuperación, consolidación y desarrollo de la agrobiodiversidad, sus tecnologías de uso y manejo y a las normas de acceso y aprovechamiento en base a sus conocimientos y prácticas, que no les son muy caras" (CONAM 2007, 61).

En el diseño de cualquier proyecto de desarrollo una de las variables más importantes es la identificación de los beneficiarios de las acciones al que van dirigidas. La antropología del desarrollo ha contribuido a la crítica sobre el uso retórico en los proyectos de términos como "participación" y "conocimientos locales", sin que ello se traduzca en un control efectivo por parte de la población (Rahnema 2012). El extracto precedente del apartado de viabilidad social del SIPAM peruano es un buen ejemplo de ello.

Replicando los principios del programa a nivel local, este proyecto persigue "revalorar los sistemas ingeniosos de gestión sostenible de los agroecosistemas de alta montaña de las comunidades originarias en el transecto Cusco y Puno como patrimonio de la humanidad" (CONAM 2007, 37). Este objetivo general se concreta en cuatro componentes específicos, a saber: a) el fortalecimiento de la "gobernanza local", entendida como "estructuras consuetudinarias ancestrales"; b) la mejora de la dieta familiar (seguridad alimentaria), a través de la implementación de tecnologías de producción agrícola (andenes, sukaqollos, qochas, bofedales, siembras asociadas y manejo de pastizales); c) la potenciación de los saberes y capacidades técnicas campesinas de manejo del clima, agua y suelo, a través de talleres dirigidos a los campe- 


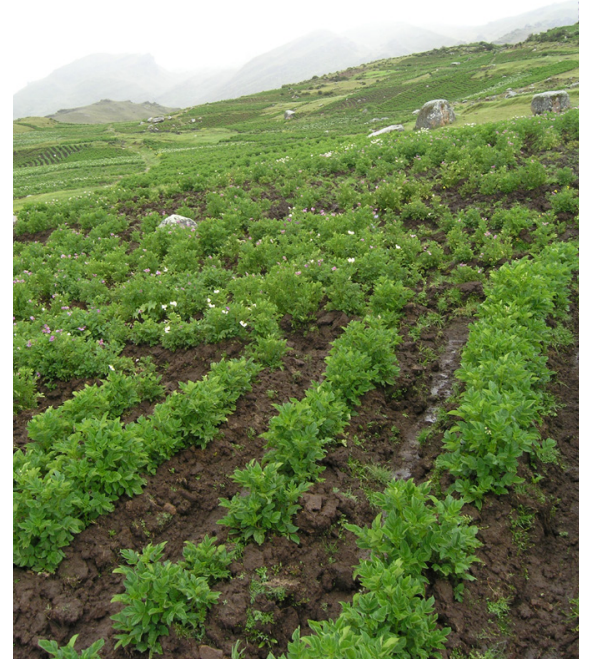

Wachu o surco de la siembra. Comunidad de Chahuaytire, Pisac, Cuzco

\section{6}

Contamos con dos versiones publicadas del proyecto, ambas elaboradas por Corredor Puno Cusco-FAO-CONAM (2006 y 2007). La de 2007 es una versión reducida (de 76 páginas) que recoge el proyecto original (de 118 páginas) elaborado en 2005 y publicado en abril de 2006. Ninguna de las dos refleja los ajustes en contenidos, duración y presupuestos realizados entre 2005 y el inicio de actividades en 2011. El documento más actualizado es un informe inédito encargado al coordinador del proyecto en marzo de 2012 (Tapia 2012). Este informe y el resto de documentos disponibles fueron contrastados mediante visitas in situ y entrevistas en pro fundidad realizadas en 2014 a una parte de la población participante del distrito de Lamay (Cuzco), y a los técnicos y gestores responsables del proyecto en Puno y en Lima, respectivamente. sinos; d) el conocimiento y la difusión científica del funcionamiento de estos agroecosistemas, mediante la elaboración de catálogos de recursos genéticos, cultivos andinos y de crianzas de alpacas de colores. No obstante, teniendo en cuenta el largo proceso de ajustes y el énfasis en los aspectos técnicos y medioambientales desde los cuales el proyecto propone la gestión del patrimonio agrícola indígena, el informe de 2012 reduce las actividades concretas a realizar durante esa campaña al último de los objetivos "registro y sistematización del conocimiento campesino sobre los principales recursos genéticos nativos en el sur del Perú (ver nota 6). Como recomienda el programa SIPAM, para visibilizar esta tarea se propone contar con la opinión y la mano de obra de los campesinos a través de sus "estructuras de organización ancestrales" en el marco de las cuales debe desarrollarse la conservación in situ de la agrobiodiversidad.

Una lectura atenta del proyecto en sus dos versiones ${ }^{6}$ arroja pobres resultados en cuanto a la identificación y caracterización de las formas de organización preexistentes en estas comunidades y su relación con los sistemas agrícolas. De hecho, en ninguna parte se explica cómo operan estas formas de organización en la toma de decisiones, en qué lugares y momentos se realizan, quiénes son las autoridades y cómo consiguen ese estatus, cómo participa en este sistema el resto de la comunidad, o cómo se establece y se significa su relación con el territorio, por mencionar solo algunos de los aspectos necesarios para viabilizar la participación local en este proyecto. En su lugar, el informe se limita a señalar la utilidad ad hoc de las formas de organización ancestrales en relación con los objetivos del proyecto: "este sistema se rige por una serie de valores y principios andinos particularmente asociados al acceso y al uso y aprovechamiento sostenible de la agro-biodiversidad en el paisaje" (CONAM 2007, 40). El bajo perfil que aparentemente presentan estas formas de autoridad tradicional en estas comunidades actualmente se justifica debido a factores externos:

"Tradicionalmente, funcionaban y funcionan en algunas comunidades los vigilantes o cuidantes de campos de cultivo en rotación sectorial, llamados arariwas y yapu campus en quechua y aimara, respectivamente. Con el proyecto SIPAM, se ha sugerido revivir y fortalecer a esas autoridades, con éxito relativo porque intervenciones externas con la creación de nuevos comités (conservacionistas, semilleristas, vaso de leche, forestación, artesanías, educación, de riego, etc.) tratan de debilitar su presencia y autoridad, con incentivos varios" (Canahua 2014, 38).

El desconocimiento sobre qué son y cómo funcionan los sistemas tradicionales de organización social y política indígenas que articulan la cosmovisión del grupo y su relación con el territorio y las prácticas agrícolas se traduce en una visión folclórica y esencialista, frecuentemente compartida por la mayoría de proyectos de desarrollo. Más allá de escuetas referencias des- 


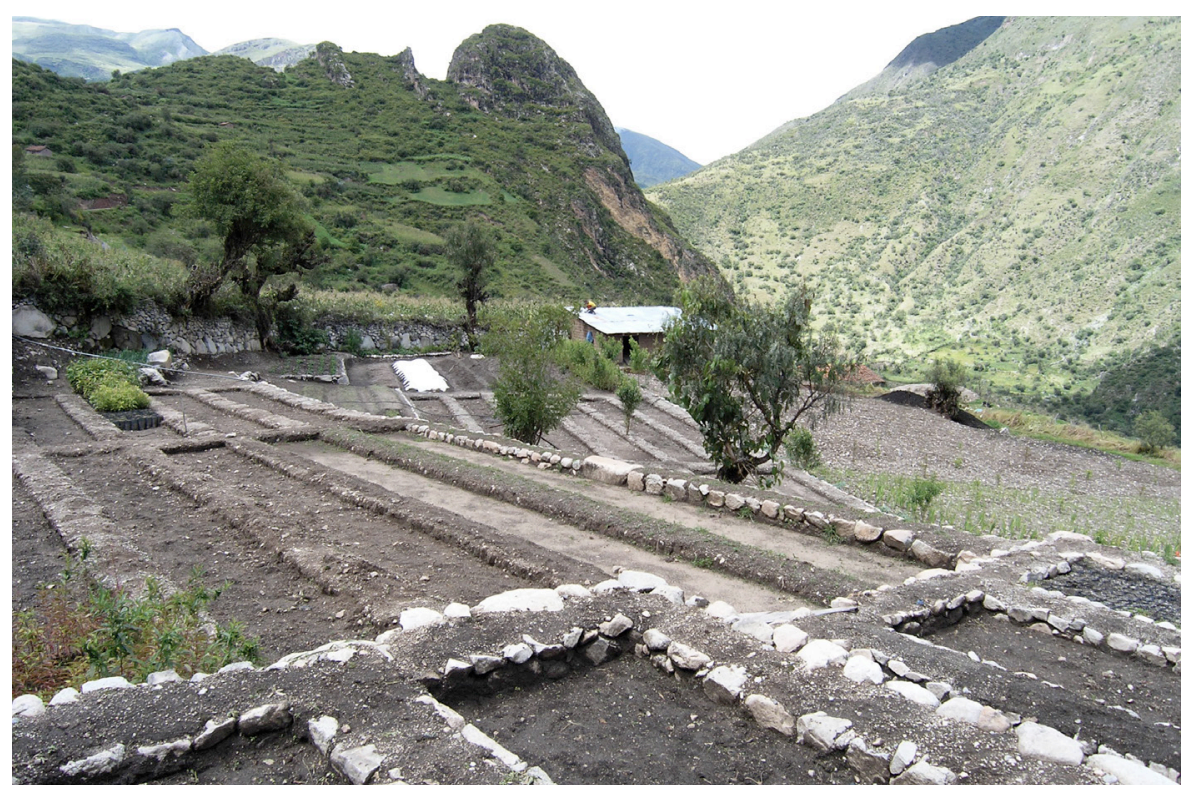

contextualizadas a los arariwas y a los alcaldes envarados (el wachu), esta visión se materializa en las actividades concretas previstas para fomentar las "estructuras ancestrales" (sic) de estas comunidades: 8 planes de gestión territorial, 8 convenios para el fortalecimiento de acuerdos comunales y, de modo singular, la celebración de 8 festivales sobre conservación dinámica de la biodiversidad agrícola.

"Canciones, música, danza, cuentos, ritos, etc. que tengan relación con las actividades agropecuarias locales y expresen mensajes y contenidos con la conservación dinámica (...) Teniendo en cuenta que el ecosistema en el mundo andino se refiere a la Pachamama donde las actividades agroproductivas se realizan en un marco lúdico, [esta actividad] se efectuará mediante festivales y concursos en cada sitio piloto y entre sitios piloto, además de que se recogerán testimonios grabados" (CONAM 2007, 40).

De hecho, si prescindimos de la narrativa sobre la conservación de la biodiversidad agrícola y la importancia otorgada sobre el papel al conocimiento indígena, nos encontramos con un conjunto de actividades dispersas, condicionadas a la definición y a los protocolos de funcionamiento del financiamiento internacional pero sin una planificación real a nivel social y cultural. Esto es, desprovistas de actores de carne y hueso y de sus relaciones sociales. Concretamente, en el SIPAM peruano estas acciones se resumen en la compra y distribución de semilla nativa entre los campesinos "conservacionistas", esto es, los participantes en el proyecto; de conos de lana de alpaca para asociaciones de tejedoras indígenas constituidas a tal efecto; de bidones de plástico para la preparación de abono orgánico líquido ("biol");
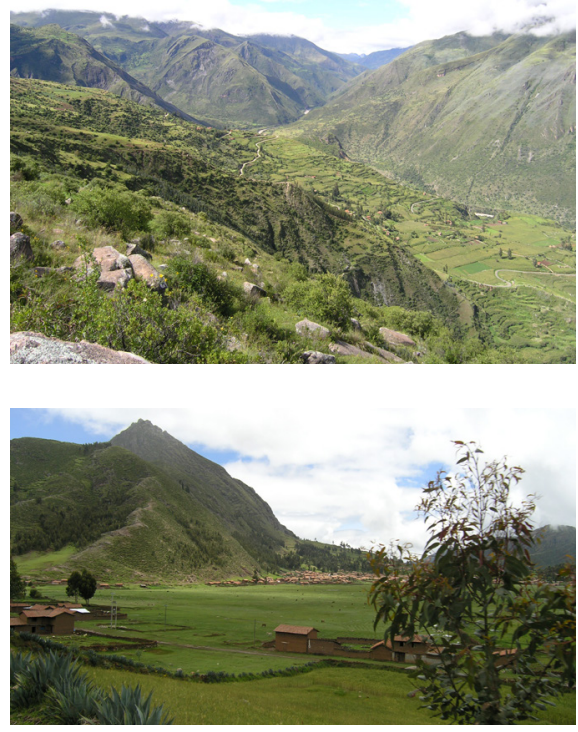

Paisajes agrícolas, distritos de Accha y Omacha, Paruro, Cuzco 
7

En un estudio clásico sobre los efectos de aparato conceptual del desarrollo en Lesoto, Ferguson (1990) concluye que más allá de los objetivos explícitos de los proyectos lo que éstos consiguen es la despolitización de los asuntos realmente relevantes para el desarrollo. la implementación de módulos de crianza de animales menores (cuyes); la construcción de almacenes rústicos de semillas de papas nativas; la organización de varias ferias locales y regionales de agro-biodiversidad para la exposición y venta de productos nativos; el acondicionamiento por parte de los técnicos de campo de varias parcelas demostrativas, y la organización de varios talleres de capacitación sobre conservación impartidos por los coordinadores del proyecto. Estas actividades y más específicamente el producto resultante de ellas donado por el proyecto constituyen para los indígenas la materialización concreta de la conservación dinámica de la agro-biodiversidad: "cuyes", "lana de alpaca", "biol" y "semillas nativas".

Llegados a este punto parece que lo que singulariza al SIPAM peruano en la práctica frente a otros proyectos de desarrollo rural en estas comunidades no es la recuperación y conservación de los sistemas agrícolas tradicionales, ni la puesta en valor de los conocimientos locales, ni tampoco el aumento de la productividad o la seguridad alimentaria. En su lugar, el efecto directo parece ser la normalización y legitimación del discurso de desarrollo, ahora "sostenible", y su introducción en las agendas nacionales. Un "aparato conceptual", al modo planteado por Ferguson $(1990)^{7}$, que en el caso de este proyecto se concreta en su contribución a la difusión del patrimonio agrícola tradicional de origen indígena de esta región como una marca que agrega valor añadido en el mercado exportador que demanda cada vez más alimentos ecológicos y de gran valor nutritivo, como se reconoce desde FAO:

"SIPAM refuerza la identidad local y nacional a través de productos y servicios únicos que [estos sitios] producen o proveen especialmente en relación con la gastronomía y el ecoturismo. Además, la marca SIPAM ofrece a los consumidores de los países en desarrollo la oportunidad de comprar estos productos y servicios para apoyar el desarrollo sostenible de los sistemas agrícolas de los que proceden. Esta estrategia es mucho más útil que comprar productos simplemente etiquetados como orgánicos o que procedan de las redes de mercado justo" (Olding y Warren 2014, 15, traducción propia).

\section{A MODO DE REFLEXIÓN FINAL}

EI SIPAM peruano, vinculado a dos de los lugares más emblemáticos a nivel natural y turístico de América Latina, es un buen ejemplo de la tendencia global en el desarrollo sostenible que concilia dos posturas aparentemente divergentes. Por un lado, la protección de paisajes muy conocidos, de gran biodiversidad cuya prioridad es la conservación y catalogación de inventarios elaborada por y para expertos y, simultáneamente, su conversión en un recurso económico orientado a mercados exclusivos como productos y servicios con un alto valor agregado por su relación con la población indígena. Un modelo de desarrollo verde en el que la dimensión ecológica y la propia 
noción de sostenibilidad son despolitizadas y despolitizadoras, y desprovistas de cultura y prácticas sociales (Arenas y Pérez Galán, 2019).

De acuerdo con Gallar y Matarán $(2015,78)$ en su estudio sobre la patrimonialización de la actividad agrícola, ese modelo que dirige el rumbo de la nueva ruralidad en las últimas dos décadas se especializa en tres sectores económicos: la agricultura industrial, la gestión de la naturaleza y el pastiche del espectáculo de la ruralidad auténtica-exótica-tradicional-indígena. En los andes peruanos estos sectores conviven con pautas estructurales de un mercado laboral y productivo que fomentan la desigualdad a través del desarrollo de fronteras étnicas entre indígenas y no indígenas, de la inversión en ciertos territorios con mayor biodiversidad y por tanto más atractivos para la conservación ambiental y el turismo, y entre campesinos pobres que constituyen la mayoría en las comunidades frente a los ejemplos exitosos de otros con mayor capital social y económico y dotes para el emprendimiento.

Por último, como ilustra el caso de la aplicación práctica de este proyecto, el discurso global del desarrollo sostenible solo trata de manera estereotipada y esencialista los sistemas de conocimiento y las formas de organización indígenas pero hace poco para confrontar la cada vez más rápida pérdida de la diversidad biológica y cultural y los derechos de uso, acceso y control de recursos y territorios de los pueblos y comunidades que habitan estos territorios (IIED et ál. 2005). Frente a esa posición es preciso avanzar en un acercamiento integral, desde una perspectiva holística, enfocado en la protección de las formas de vida y los derechos humanos de los pueblos indígenas no solo como proveedores de productos ecológicos para satisfacer las modas de consumo de mercados globales. Pero, en tal caso, es muy probable que no se llame "desarrollo". 


\section{BIBLIOGRAFÍA}

- Altieri, M. A. (1999) Agroecología. Bases científicas para una agricultura sustentable. Montevideo: NordanComunidad

- Arenas, M.A. y Pérez Galán, B. (2019) Naturaleza, conservación e identidad verde en Costa Rica. Antropología Experimental, n. ${ }^{0}$ 19. Disponible en: https:// revistaselectronicas.ujaen.es/index.php/rae/article/ view/4912 [Consulta: 22/04/2021]

- Calavia, O. (2006) El indio ecológico. Diarios a través del espejo. Revista de Occidente, n. ${ }^{\circ} 298$, pp. 27-42

- Canahua, A. (2014) Informe de Evaluación Final del Proyecto Sistemas Importantes del Patrimonio Agrícola Mundial. Informa inédito

- Castillo, J. (dir.) (2013) Carta de Baeza sobre Patrimonio Agrario. Sevilla: Universidad Internacional de Andalucía. Disponible en: https://www.unia.es/explorar-catalogo/item/ carta-de-baeza [Consulta: 07/07/2021]

- Castillo, J. y Martínez, C. (coords.) (2015) Patrimonio Agrario. La construcción cultural del territorio a través de la actividad agraria. Sevilla: Universidad Internacional de Andalucía. Disponible en: https://dspace.unia.es/ bitstream/handle/10334/3525/2015 978-84-7993-264-0 patrimonioagrario.pdf?sequence $=1$ [Consulta: 28/08/2021]

- cONAM [Consejo Nacional del Ambiente] (2006) Proyecto: Sistemas ingeniosos del patrimonio agrícola mundial Corredor Puno-Cusco. Resumen ejecutivo. Consejo Nacional del Ambiente-CONAM, Fao. Lima, abril de 2006. Disponible en: https://repositoriodigital.minam gob.pe/handle/123456789/107 [Consulta: 28/04/2021]

- CONAM [Consejo Nacional del Ambiente] (2007) Proyecto: Sistemas Ingeniosos del Patrimonio Agrícola Mundial. Corredor Puno-Cusco. Resumen ejecutivo. Consejo Nacional del Ambiente-CONAM, Fao. Lima, septiembre de 2007

- CONAM-INIA [Consejo Nacional del Ambiente-Instituto Nacional de Investigación Agraria] (2003) Documento base del Programa Nacional de Agrobiodiversidad

- Connerton, P. (1989) How societies remember. Cambridge: Cambridge University Press

- Cosgrove, D. (1984) Social formation and symbolic landscape. New Jersey: Barnes \& Noble Books

- Descola, P. (2001) Construyendo naturalezas. Ecología simbólica y práctica social. En: Descola, P. y Pálsson, G. Naturaleza y Sociedad. Perspectivas antropológicas. México: Siglo XXI, pp. 101-123

- Erickson, C.L. (2006) El valor actual de los camellones de cultivo precolombinos: experiencias del Perú y Bolivia En: Valdez, F. (ed.) Agricultura ancestral. Camellones y albarradas. Contexto social, usos y retos del pasado y del presente. Quito-Ecuador: Ediciones Abya-Yala, pp. 315339

- Escobar, A. (1999) El lugar de la naturaleza y la naturaleza del lugar: globalización o posdesarrollo. En: Viola, A. (comp.) Antropología del desarrollo. Teorías y estudios etnográficos en América Latina. Barcelona: Paidós Studio, pp. 169-216

- FAO [Organización de las Naciones Unidas para la Agricultura y la Alimentación] (2008) Conservation and Adaptive Management of Globally Important Agricultural Heritage Systems (GIAHS). Terminal Report. Project ID: 137561 Disponible en: http://www.fao.org/fileadmin/ templates/giahs/PDF/GIAHS_B_terminalReport.pdf [Consulta: 27/05/2021]

- Ferguson, J. (1990) The Anti-politics machine: "Development", Depoliticization, and bureaucratic power in Lesotho. Nueva York: Cambridge University Press

- Gallar. D. y Mataran, A. (2015) La construcción social de la ruralidad. Coevolución, sustentabilidad y patrimonialización. En: Castillo, J. y Martínez, C. (coord.) (2015) Patrimonio Agrario. La construcción cultural del territorio a través de la actividad agraria. Sevilla: Universidad Internacional de Andalucía, pp. 73-117

- Hecht, S. (1999) La evolución del pensamiento agroecológico. En: Altieri, M. (ed.) Agroecología. Bases científicas para una agricultura sustentable. Montevideo: Nordan-Comunidad. pp. 1-14

- Herrera Wassilowsky, A. (2011) La recuperación de tecnologías indígenas. Arqueología, tecnología y desarrollo en los Andes. Lima-Bogotá: Instituto de Estudios PeruanosUniversidad de Los Andes

- Herrera Wassilowsky, A. (2013) Arqueología y desarrollo en el Perú. En: Herrera Wassilowsky, A. (comp.) Arqueología y Desarrollo en América del Sur. Bogotá-Lima: Instituto de Estudios Peruanos-Universidad de los Andes, pp. 75-93

- Hirsch, E. (1995) Landscape: between place and space. En: Hirsch, E. y O'Hanlon, M. (ed.) The anthropology of landscape. Perspectives on place and space. Oxford: Clarendon, pp. 1-30

- Howard, P., Puri, R., Jane Smith, L. y Altierri, M. (2008) Globally Important Agricultural Heritage Systems: A Scientific Conceptual Framework and Strategic Principles. Roma: FAO. Disponible en: http://www.fao.org/3/ap025e/ ap025e.pdf [Consulta: 06/07/2017]

- IIED [Instituto Internacional para el Medio Ambiente y Desarrollo], Fundación Dobbo-Yala, Universidad de Panamá, Centro Chino de Política Agrícola, Instituto de Investigación Políticas del Medio Ambiente y Agricultura del Sur, Kenya Instituto de Investigación Forestal, Centro para Sistemas de Cultivos Indígenas, Ecoserve y Centro 
de Investigación Herbal y del Folclore (2005) Protección de Conocimiento Tradicional y Patrimonio Culturalel Concepto del 'Patrimonio Bio-Cultural Colectivo'. Grupo de Trabajo de Poblaciones Indígenas, Sesión 23. Disponible en: https://pubs.iied.org/es/g01068 [Consulta: 02/05/2021]

- Koohafkan, P. y Altieri, M. (2010) Sistemas importantes del Patrimonio Agrícola Mundial. Un legado para el futuro. Roma: FAO

- Küchler, S. (1993) Landscape as memory: the mapping of process and its representation in a Melanesian Society. En: Bender, B. (ed.) Landscape. Politics and perspectives. Oxford: Berg, pp. 85-106

- Lakoff, G. y Johnson, M. (1995) Metáforas de la vida cotidiana. Valencia: Cátedra

- Moose D., (2012) Una etnografía de las políticas de la ayuda en la práctica. En: Pérez Galán, B. (ed.) Antropología y Desarrollo. Discurso, Práctica y actores. Madrid: La Catarata, pp. 258-281

- Olding W. y Warren, P. (2014) Conservation and Adaptive Management of Globally Important Agricultural Heritage Systems (GIAHS). Project-GCP/GLO/212/ GFF. Final evaluation of the GIAHS initiative. Roma: FAO

- Pérez Galán, B. (2004) Somos como Incas. Autoridades tradicionales en los Andes peruanos. Madrid- Frankfurt: Iberoamericana-Vervuert

- Pérez Galán, B. (2012) (ed.) Contribuciones antropológicas al desarrollo. En: Pérez Galán, B. (ed.) Antropología y Desarrollo. Discurso, Práctica y actores. Madrid: La Catarata, pp. 18-36

- Pérez Galán, B. (2015) Discursos globales y prácticas locales sobre agrobiodiversidad y conocimientos tradicionales en comunidades indígenas. El programa SIPAM en el sitio piloto de Machu Picchu al Lago Titicaca (Perú). En: Castillo, J. y Martínez, C. (coords.) (2015) Patrimonio Agrario. La construcción cultural del territorio a través de la actividad agraria. Sevilla: Universidad Internacional de Andalucía, pp. 231-267

- Pérez Galán, B. (2017) Desarrollo sostenible y usos del patrimonio agrícola en los Andes. En Tomé, P. (ed.) Reflexiones Rayanas. Vol. II. Ávila: Asociación de Antropología de Castilla y León "Michael Kenny", pp. 3-30

- Ploeg, J. (1990) Sistemas de conocimiento, metáfora y campo de interacción: el caso del cultivo de la patata en el altiplano peruano. Agricultura y Sociedad, n. ${ }^{\circ}$ 5, pp. 143-166

- Rahnema, M. (2012) Participación. En: Pérez Galán, B. (ed.) Antropología y Desarrollo. Discurso, Práctica y actores. Madrid: La Catarata, pp.187-207
- Reyes-García, V. (2007) El conocimiento tradicional para la resolución de problemas ecológicos contemporáneos. Papeles, n. ${ }^{\circ} 100$, pp. 109-116

- Tapia, M. (1997) Los sistemas de producción agrícola campesina en los Andes del Perú. En: La sostenibilidad de los sistemas de producción campesina en los andes. Lima: CONDESAN

- Tapia, M. (2012) Consultoría Proyecto SIPAM-Perú. Informe inédito

- Toledo, V. (1992) What is ethnoecology? Origins, scope, and implications of a rising discipline. Etnoecologica, n. ${ }^{0} 1$, pp. 5-21

- Viveiros de Castro, E. (2014) La mirada del jaguar. Introducción al perspectivismo amerindio. Entrevistas. Buenos Aires: Tinta Limón

- Warren, D.M., Slikkerveer, J.L. y Brokensha, D. (ed.) (1995) The cultural dimension of development: Indigenous knowledge systems. London: Intermediate Technology Publications 